\title{
LAS RELACIONES INTERGUBERNAMENTALES (RIG) HERRAMIENTA FUNDAMENTAL PARA LA DESCENTRALIZACIÓN Y REFORZAMIENTO DE LA GOBERNANZA
}

\author{
Claudia Marcela Rodríguez R. ${ }^{1}$
}

\section{Resumen}

El artículo pretende acercarnos a tres conceptos de suma importancia cuando se habla de gestión y políticas públicas, estos conceptos como son las relaciones intergubernamentales, la descentralización y la Gobernanza, cada vez vienen cobrando más protagonismo al hablar del mejoramiento en la gestión pública, encaminada a una gestión plural, diversa y participativa. Estos aspectos son los que se estudian a lo largo del artículo así como su aplicación en el caso Colombiano.

Palabras Clave: Descentralización, políticas públicas, Gobernanza, coordinación, Gestión

INTERGOVERNMENTAL RELATIONS (IGR). Essential tool for decentralization and strengthening governance

\section{Abstract}

The article aims to approach three important concepts when talking about public policy and management, these concepts such as intergovernmental relations, decentralization and governance are increasingly gaining more importance to discuss improvement in governance, aimed at management plural, diverse and participatory. These aspects are being studied throughout the paper and its implementation in the Colombian case.

Keywords: decentralization, public policy, governance, coordination, management

\footnotetext{
${ }^{1}$ Politóloga Universidad Nacional de Colombia, Máster en Administración y Gerencia Pública 


\section{Introducción}

En los últimos años los estudios de descentralización y de relaciones intergubernamentales (RIG) han ido cobrando gran importancia a través de los procesos de democratización, integración y de la importancia que se le ha venido dando cada vez más a los entes territoriales.

El tema de la política de descentralización ha sido objeto de numerosos estudios tanto en Europa como Latinoamérica, teniendo en cuenta su impacto a nivel ad intra, y en su nivel externo, y la importancia que ello tiene para el fortalecimiento de la gobernabilidad y Gobernanza institucional. De ahí la importancia del fortalecimiento de un proceso descentralizado coherente y exitoso, que lleve a una mayor profundización de la Gobernanza, entendida ésta como un proceso de coordinación de actores, grupos sociales, instituciones; que permitan lograr metas definidas colectivamente en entornos fragmentados y caracterizados muchas veces por la incertidumbre.

Por ello la coherencia de la acción pública (la definición de problemas, la toma de decisiones y su ejecución) no pasa por la acción aislada de una elite político-administrativa relativamente homogénea y centralizada, sino por la adopción de formas de coordinación a distintos niveles y multifactorial (Legales, 1998). En este marco lo que pretendo mostrar a lo largo del artículo es un acercamiento a conceptos básicos e importantes de la gestión pública, relaciones intergubernamentales, la descentralización y la Gobernanza, cada vez vienen cobrando más protagonismo al hablar del mejoramiento en la gestión pública, encaminada a una gestión plural, diversa y participativa. Estos aspectos son los que se estudian a lo largo del artículo así como su aplicación en el caso Colombiano; en donde es evidente la necesidad del fortalecimiento institucional de las entidades regionales y locales, que velen por responder a esa necesidad de un Estado comprometido con una gestión plural, diversa, participativa y donde se ofrezcan instancias de toma decisiones mediada por diferentes actores y que realmente responda a las necesidades de los diferentes colectivos que componen nuestra gran riqueza cultural, social y de género. En este marco lo que pretende el artículo es ofrecer una reflexión de la importancia de configuración y del fortalecimiento de las instancias intergubernamentales que permiten el fortalecimiento de la gobernanza y la configuración de una mayor descentralización que responda a las necesidades de las diferentes comunidades locales, ofreciendo un mayor acercamiento de la administración a las ciudadanas y ciudadanos de nuestro país, ello implica el fortalecimiento de cuadros y de gerentes y gestores públicos al servicio de la ciudadanía que respondan a la necesidad de generar cuadros institucionales fortalecidos y comprometidos con el desarrollo democrático y participativo.

\section{Descentralización}

El soporte conceptual y empírico de la descentralización proviene principalmente de la ciencia política, de la economía y de la administración. Para comprender la descentralización es necesario tener en cuenta los fines que la motivan, entre ellos la redistribución del poder, el fortalecimiento de la democracia, acercar las decisiones a los ciudadanos, y mejorar la eficiencia entre otros. Boisier (1989) Distingue tres formas de descentralización: la funcional, la territorial (ambas hacia autoridades designadas) y la política («cuando el cuerpo descentralizado se genera mediante procesos electorales») (Boisier, 1990).

Una definición más técnica, la describe como un proceso mediante el cual se transfiere poder de decisión y responsabilidades desde el nivel central a otros niveles territoriales, ya sea de orden regional, y/o local, para la gestión de sus asuntos. Supone el traspaso de funciones, competencias y recursos con miras al cumplimiento de los objetivos, principalmente el de mejorar 
la calidad de vida de los ciudadanos, y vincular a los ciudadanos como sujetos activos en el lineamiento y toma de decisiones (Planeación Nacional, 2008).

De igual modo la descentralización supone el fortalecimiento de la autonomía de los órganos territoriales, y el desarrollo de la cultura política y administrativa. El concepto de autonomía (se entiende el derecho y la capacidad efectiva de las entidades territoriales de ordenar y gestionar una parte importante de los asuntos públicos, en el marco de la ley, bajo su propia responsabilidad y en beneficio de sus habitantes) tiene una especial importancia cuando hablamos de descentralización. La autonomía local debe ser real y operativa, debe comprender no solo la capacidad normativa, reguladora y decisoria de los entes locales, sino capacidad de gestión y la definición de estrategias, objetivos y políticas (Carta Europea local, 1998). La autonomía local debe ser real y operativa, debe comprender no solo la capacidad normativa, reguladora y decisoria de los entes locales, sino capacidad de gestión y la definición de estrategias, objetivos y políticas (Arana, 2007).

\section{Descentralización y participación}

Algunos teóricos parten de la hipótesis de que a mayores niveles de descentralización mayores serán los niveles de participación ciudadana, ya que se acerca el poder y la toma de decisiones al ciudadano, por tanto hay una ampliación del espectro de toma de decisiones a otros actores.

En este marco; por un lado, la descentralización se ve como un instrumento para la democratización del Estado y la promoción de la participación ciudadana en la definición y fiscalización de las políticas públicas. Por otro, se equipara la descentralización a la disminución del papel del Estado como ente de regulación y redistribución de recursos y se promueve la participación ciudadana exclusivamente en la ejecución de acciones definidas en el nivel central o en térmi- nos de complementariedad en la prestación de servicios públicos por parte de actores privados, equiparando el «ciudadano» al «cliente».

Según North estimular el involucramiento ciudadano en la gestión pública es ciertamente un objetivo político en sí, propio de la democratización, pero del análisis anterior se desprende que la participación resulta además un requisito indispensable para activar mecanismos de eficiencia en la gestión pública, y del fortalecimiento de la legitimación democrática y de la Gobernanza institucional- El proceso descentralizador permite que la administración este mas cercana al usuario (Olías de Lima,2008), de allí, que permitirá un fortalecimiento de la Gobernanza, y con ello una profundización del mismo proceso descentralizador, pero es evidente que todo este proceso pasa por principios básicos de legitimidad y eficacia (tomar decisiones informadas y justas que respondan adecuadamente a las demandas de la ciudadanía).

De esta manera es evidente que a consecuencia de la multiciplicidad de instancias, niveles y actores que intervendrán en la gestión es posible que las tensiones aumenten, esto implica que las administraciones tienen que tener nuevas habilidades, pero también y sobre todo que necesitan reforzar su legitimación, y tener una relación mas próxima al ciudadano, esto se logra mediando la implementación de un proceso descentralizador que permita el acercamiento de la administración a las necesidades de los ciudadanos, todo ello teniendo en cuenta la cada vez mas existencia de entornos regionales diversos y multiculturales y con enfoques sectoriales (Género, étnico, entre otros) (Olías de Lima,2008).

De otro lado según José Carlos Illán (2007), la participación es uno de los canales básicos para un proceso descentralizador; es así que el autor identifica, por lo menos, dos factores a la hora de emprender el diseño de cualquier 
programa descentralizador para cualquier país en desarrollo: el primero tiene que ver con la institucionalización de los canales de participación que existan y el segundo con la habilidad (aunque algunos también añadirían capacidad) de la población para utilizarlos.

\section{Gobernanza}

Autores como Prats (2002) y Cruz (2001) asumen la Gobernanza como «un concepto amplio que engloba el conjunto de actores, valores e instituciones que vinculados de distintas maneras conforman la red que da soporte al conjunto del sistema social y político, marco en el que el gobierno llega a constituirse en un actor más, que se relaciona e interactúa con el resto en base a sus propias capacidades (gobernabilidad)» (Arana, 2007). Igualmente la Gobernanza es entendida como un nuevo estilo de gobierno, basada en los principios de transparencia, participación y responsabilidad, que difiere de los estilos tradicionales de control jerárquico y también es diferente en cuanto al papel que otorga a la interacción y la cooperación entre los poderes públicos y los actores no estatales en el interior de redes decisionales mixtas entre lo público y lo privado (Mayntz, 2000), de esta manera evidenciamos la importancia que tiene los mecanismos de coordinación o de relaciones intergubernamentales por un lado para el establecimiento de la descentralización, pero también para el fortalecimiento de la Gobernanza, legitimidad institucional a través de la voz y voto de diferentes actores.

La Gobernanza, al establecerse como una condición de la totalidad del sistema político, parte de un concepto mucho más amplio que, incluyendo al propio gobierno, se relaciona con el conjunto de «patrones y estructuras mediante las cuales los actores políticos y sociales llevan adelante procesos de intercambio, coordinación, control, interacción y toma de decisiones, dentro y entre órdenes sociales y regimenes democráticos, y que en su dimensión prescriptiva-normativa, busca en la actualidad ser consecuente con los valores de la democracia» (Cruz, 2001), bajo este concepto la Gobernanza por tanto es la base indiscutible para explicar el proceso de configuración de las relaciones intergubernamentales, y por tanto es el concepto que se abordará en el trabajo.

Dentro de este marco podemos especificar que el concepto de Gobernanza no es nuevo. Es tan antiguo como la civilización humana. De esta manera identificamos que la Gobernanza como concepto aislado significa: el proceso de toma de decisiones y el proceso por el que las decisiones son implementadas, o no. ${ }^{2}$ El término Gobernanza puede ser utilizado en diferentes contextos, como por ejemplo Gobernanza corporativa, Gobernanza internacional, Gobernanza nacional y Gobernanza local.

Dado que la Gobernanza es el proceso de toma de decisiones y el proceso por el que estas son implementadas, o no, el análisis de la Gobernanza se centra en los actores, formales e informales, que están involucrados en el proceso de toma de decisiones y en su implementación, así como en las estructuras, formales e informales, que se han preparado para poder implementar las decisiones (relaciones intergubernamentales).

Camou, unificando criterios, la define como un estado dinámico de equilibrio en tensión entre el nivel de las demandas societales y la capacidad del sistema de poder público de responderlas de manera legítima y eficaz, entendiendo que el cada vez más complejo escenario social tiende a generar, principalmente en los países de América Latina, demandas específicas que exigen de una mayor capacidad política en la estructuración y aplicación de soluciones adecuadas a los problemas colectivos (Camou, 2001).

\footnotetext{
Texto original de la UN ESCAP, Comisión Económica y Social de las Naciones Unidas para Asia y el Pacífico. www.unescap.org
} 
De otro lado Patrick Le Galès (1998) resume estas preocupaciones al insistir en la complejidad de las nuevas formas de interacción entre actores de las políticas públicas. Para él, «se encuentran en la Gobernanza las ideas de dirección de gobierno sin otorgar primacía al Estado. Plantear la cuestión de la Gobernanza sugiere la comprensión de la articulación de los diferentes modos de regulación de un territorio, a la vez en términos de integración política y social y en términos de capacidad de acción».

\section{Papel de los actores en la configuración de la Gobernanza}

En estas condiciones, la Gobernanza aparece como una forma de gobierno (en su sentido mas amplio) en la que la coherencia de la acción pública (la definición de problemas, la toma de decisiones y su ejecución) no pasa por la acción aislada de una elite político-administrativa relativamente homogénea y centralizada, sino por la adopción de formas de coordinación a distintos niveles y multiactorial, cuyo resultado, siempre incierto, depende de la capacidad de los actores públicos y privados para definir un espacio común, de su capacidad para movilizar expertos de orígenes diversos y de implantar modos de responsabilización y de legitimación de las decisiones.

Así, los espacios políticos supranacionales se revelan como escenarios privilegiados en los que se desarrollan estas nuevas formas de acción pública que pondrían de manifiesto la transformación de las formas de representación en las sociedades complejas y, en particular, la tendencia a la ruptura entre la esfera de las políticas públicas y la esfera de la representación política (en el sentido de la constitución de un vínculo social de pertenencia entre el individuo y la sociedad), lo que implica una mayor opacidad e incertidumbre en los sistemas de representación de intereses. (Ruano de la Fuente, 2002). Según Olías de Lima y Del
Campo se han constituido nuevas formas de Gobernanza. Se podría decir que más allá de las diferencias, la Gobernanza hace referencia a la importancia que han cobrado actores e instituciones no estatales en el proceso de decisión pública, e incluso, de dirección política. Partiendo de este reconocimiento de la multiplicidad de intereses y actores, que quiebra la drástica distinción entre lo público y lo privado, la mayor parte de los autores reconocen la existencia de redes más o menos formalizadas en diferentes ámbitos políticos y administrativos: En las relaciones intergubernamentales, como Rhodes; en la arena transnacional como Rosenau, o en el contexto de las teorías de la democratización y del desarrollo como Hyden, para todos estos autores la Gobernanza moderna es simplemente un nuevo modo de gobernar en el que las instituciones estatales y no estatales, los actores públicos y privados, participan y, a menudo, cooperan en la formulación y la aplicación de las nuevas políticas públicas (Le Galès, 1998).

De otro lado la presencia del Estado en los procesos de Gobernanza puede ir desde su contemplación como coordinadores hasta ser uno tan sólo de los actores políticos más relevantes del proceso de Gobernanza. Una de las cuestiones centrales consiste en llegar a saber en qué medida la Gobernanza altera el poder y las capacidades del Estado. La emergencia de nuevas formas de gestión pública basadas en la colaboración público-privada, la actuación de las organizaciones del tercer sector o las modificaciones inducidas por organizaciones supranacionales o transnacionales ponen en tela de juicio la configuración institucional del Estado y las formas de gestión de sus instituciones. (Le Galès, 1998). De allí la importancia del reforzamiento y coordinación Institucional y de las relaciones Intergubernamentales. 


\section{Relaciones intergubernamentales}

Este término se origino en los Estados Unidos durante el decenio de 1930, para explicar las relaciones de los entes estatales federados de este país.

Las relaciones intergubernamentales o espacios de coordinación (en adelante RIG) se configuran como en instrumento importante para la construcción de enramados institucionales, que además de una mayor descentralización implique la articulación y la cooperación efectivas entre las instancias nacional, regional y local, para beneficio de la vida y las instituciones del país de manera vertical y una interrelación horizontal entre diferentes instancias para la implementación de políticas públicas.

Anderson (1960) define las RIG como un cuerpo de actividades o interacciones que ocurren entre unidades gubernamentales de todas clases y niveles del sistema. Para Wright (1997), este término no es un mero sustituto del federalismo, especificándolo, como que las RIG trascienden las relaciones entre nación y Estados, así como los aspectos meramente legales y las relaciones jerárquicas; además implican un enfoque empírico y orientado a resultados de las políticas públicas. A continuación se trata de responder claramente que es como y en que ámbitos actúan:

¿Quién? Las RIG incluyen a la vez a los ciudadanos y a funcionarios públicos, así como a entidades gubernamentales de todos los tamaños, tipo y ubicaciones.

¿Donde? Se encuentran por doquier en nuestro sistema político y administrativo, las RIG están presentes en todo el ámbito gubernamental.

¿Cuando? LAS RIG son actuales y enfocan cuestiones críticas de las políticas públicas; pero las RIG también tienen hondas raíces en el pasado y tendrán consecuencias importantes sobre cómo nos enfrentamos en el futuro a problemas como la educación, el ambiente, la salud, el transporte y demás.

¿Que? William Anderson (1960) las definió como un importante cuerpo de actividades o de interacciones que ocurren entre las unidades gubernamentales de todos tipos y niveles dentro del sistema. Caracterizada por el modelo de autoridad superpuesta, presenta a las RIG como una conducta pautada interdependiente y negociada entre funcionarios nacionales, estatales y locales. Los contactos y los intercambios entre funcionarios pueden ser simultáneamente cooperativos y competitivos; los factores determinantes pueden incluir la cuestión o problema de política, la categoría de los funcionarios (electos o nombrados); las inclinaciones partidistas de los participantes y el electorado (local, estatal, o nacional), representado. Este modelo de autoridad superpuesta es la que se adapta mejor a los acuerdos negociados y a la propensión a la disputa, que caracterizan a las RIG contemporáneas.

Igualmente Anderson la tipifica como «un importante contingente de actividades 0 interacciones que tienen lugar entre unidades de gobierno de todo tipo y nivel territorial de actuación» (Wright, 1997).

\section{Caracterización de las relaciones intergubernamentales}

Para hacer una mejor explicación de este concepto y lo que configuran las relaciones intergubernamentales nos remitiremos a Robert Agranoff (1995) que ha sido un gran teórico y estudioso del tema. En su perspectiva, el interés por esta materia se inicia a finales de la década de los años setenta como una superación de los análisis tradicionales acerca de los sistemas unitarios y federales, muchos estudios empezaron a hacer referencia al tema, entre ellos los de Kenneth Hanf y Fritz Scharpf (1979), que contemplaron el proceso de implantación de 
distintas políticas públicas desde una perspectiva intergubernamental, haciendo especial hincapié en los limites de las posibilidades de coordinación y control de las mismas.

El gobierno central fija a menudo las condiciones de prestación o establece los parámetros de los servicios pero no los administra. El resultado, la implicación de diversas instituciones pertenecientes a distintos niveles de gobierno, nos sugiere la existencia de una situación de interdependencia. En resumen, la actuación pública implica la presencia de una dimensión territorial en el ejercicio de la autoridad junto al desempeño de determinadas responsabilidades funcionales. La situación de interdependencia antes enunciada da lugar a las relaciones intergubernamentales. La cuestión crucial acerca de estos entramados organizativos es según sugiere Rose (1995), la relativa a sus mecanismos de interrelación, y que entre los modelos posibles parece ser el intergubernamental/ínter organizativo, el que más se ajusta a los cambios que se están presentando en la realidad.

Wright Deils establece cinco rasgos distintivos de las relaciones intergubernamentales: Como primer aspecto se resalta que las relaciones intergubernamentales trascienden las pautas de actuación gubernamental constitucionalmente reconocidas e incluyen una amplia variedad de relaciones entre el nivel nacional y el local, entre el nivel intermedio (estatal, regional o provincial) y el local, y/o entre las distintas unidades de gobierno local.

El segundo rasgo de las relaciones intergubernamentales es la importancia del elemento humano: las actividades y actitudes del personal al servicio de las unidades de gobierno. Wright sostiene que, en sentido estricto, «no existen relaciones entre gobiernos, únicamente se dan relaciones entre las personas que dirigen las distintas unidades de gobierno».
Como tercer elemento se establece que las relaciones entre empleados públicos suponen un continuo contacto e intercambio de información y puntos de vista. Wright opina que una de las preocupaciones centrales de los participantes en las RIG es la de conseguir que los asuntos «salgan adelante», propósito que se alcanza a través de diversos mecanismos informales, prácticos y orientados a fines concretos, desarrollados dentro del marco formal y legal en que se devuelven los distintos actores. Por otra parte es bien sabido que las reglamentaciones, procedimientos y directrices de interpretación que acompañan a la normativa y a las subvenciones intergubernamentales son parte de los medios usuales de intercambio entre los distintos niveles de gobierno.

El cuarto rasgo señalado por Wright consiste en que cualquier tipo de empleado público es, al menos potencialmente, un participante en los procesos intergubernamentales de adopción de decisiones. Los actores implicados proceden no sólo de los diferentes niveles territoriales, sino también de los distintos poderes: legislativo, ejecutivo y judicial. En España por poner un ejemplo, el parlamento ha actuado como instrumento para limar asperezas en la definición del papel de las comunidades Autónomas contempladas en la constitución de 1978. Por su parte el Tribunal Constitucional, además de tener jurisdicción sobre los temas relacionados con la capacidad legislativa y reglamentaria, decide en caso de conflicto de competencias entre el Estado y las Comunidades Autónomas.

Como último y quinto elemento encontramos que las RIG se caracterizan por su vinculación a las políticas públicas. Wright entiende que esta dimensión hace referencia al conjunto de intenciones y acciones (o no acciones) de los empleados públicos, así como a sus consecuencias, lo cual nos obliga a examinar las interacciones de los distintos actores en las fases de formulación, implementación y evaluación de 
las políticas. El concepto de RIG trasciende los enfoques constitucionalmente legales acerca tanto de las divisiones entre gobiernos como de sus respectivas funciones.

\section{Relaciones intergubernamentales y diseños de la descentralización Pluralismo institucional y monopolio institucional}

El diseño institucional en un país puede adaptar distintas modalidades, con relación a los papeles políticos y organizativos necesarios para llevar a cabo las tareas que encara el sector público. Siguiendo la clasificación de Cohen y Peterson (2007), encontramos áreas de políticas públicas en las que predomina el monopolio institucional, otras donde se establece un monopolio institucional distribuido, y finalmente, otras en las que existe pluralismo institucional. El monopolio institucional se refiere a aquellos papeles que el Estado mantiene en exclusividad para sus órganos y departamentos centrales. El monopolio institucional distribuido se refiere a aquellas responsabilidades que han sido transferidas completamente a órganos de gobierno subnacional en el territorio (local, estatal, agencias, etc.) mediante alguna de las fórmulas de descentralización. Estos órganos mantienen el monopolio en su ámbito territorial o sectorial, reproduciendo la misma lógica de concentración sobre las tareas asignadas en sus instituciones y organizaciones específicas. En cuanto al pluralismo institucional los autores evidencian que aparece cuando en un proceso de descentralización, las responsabilidades sobre determinadas tareas son compartidas por más de una institución u organización, que pueden pertenecer a niveles de gobierno o ámbitos territoriales distintos, participando incluso organizaciones públicas no estatales o el sector privado (Aja, 1999). Las situaciones de pluralismo institucional tienden a producir una compleja estructura de interdependencias entre los actores implicados en cada política pública, apareciendo por tanto unas relaciones intergubernamentales más intensas (Jordana, 2001).

\section{Hacia el Fortalecimiento de Instancias de las RIG en Colombia}

Se han llevado a cabo muy pocos estudios en el tema de las relaciones intergubernamentales en Colombia, sin embargo a nivel internacional en el marco de instancias supranacionales encontramos un gran desarrollo teórico al respecto y que dan cabida para aplicar y analizar ciertas categorías de la gobernanza de un escenario internacionales, en la gobernanza local (El caso de la Unión europea). Colombia tiene un precario desarrollo institucional y de normativa para la regulación de instancias intergubernamentales; de igual modo podemos identificar esa falta de interés en el tema o de estudio de éste aspecto, debido a que muchos diseños de descentralización han sido copiados de otros países con mayor nivel de desarrollo, sin tener en cuenta las tradiciones políticas locales, la capacidad técnica o reglamentaria de cada país, u otros muchos factores específicos de la región. De otro lado también puede influir que, en muchos casos, numerosas lógicas sectoriales -desconcentradoras o descentralizadoras- con criterios distintos, se han superpuesto en los mismos países sin que se asentara antes un proceso de reestructuración de la legitimidad política entre los distintos niveles gubernamentales. De allí la importancia de crear modelos propios, auténticos que respondan a nuestra idiosincrasia, cultura, economía y que brinde soluciones propias para problemas propios de una sociedad que viene sufriendo más de 50 años de un conflicto armado interno y que necesitará de instancias gubernamentales más fortalecidas con una mayor participación social en instancias locales, que permitan tomar decisiones inclusivas en beneficio de la comunidad.

Todo proceso de descentralización territorial acometido por un Estado inicialmente unitario, se emprende con una preocupación esencial: lograr que los ámbitos de autonomía que se reconozcan a los nuevos entes no pongan en peligro la «unidad organizada de decisión y acción» en que el propio Estado consiste (Heller, 1985). 
La legislación dictada y la jurisprudencia relacionada con el tema, han privilegiado el principio de la unidad política del Estado, neutralizando el tránsito hacia un modelo territorial autonómico esbozado a muy grandes rasgos por el texto constitucional. Lo que explica también, en cierta medida, las dificultades que han tenido las distintas propuestas de fortalecimiento de los procesos de descentralización política para abrirse camino (Restrepo, 2006). Ante un Estado Unitario y, por ende, centralizado políticamente, pero con descentralización administrativa, debe recalcarse que dicha descentralización y autonomía tienen limitaciones de orden constitucional, que se desprenden, para comenzar, de la propia adopción de la forma unitaria por la Carta Política una de cuyas primeras consecuencias es, como se ha dicho, la de la uniformidad legislativa en el ámbito nacional, de esta manera en el territorio colombiano podrían organizarse hasta seis clases de entidades territoriales ${ }^{3}$. De forma que cabría la posibilidad de que existiera entre el nivel nacional y el local hasta tres niveles intermedios de gobierno -las provincias, los departamentos y las regiones-, pluralidad que aún no siendo ajena en Derecho Comparado, pudiera resultar totalmente disfuncional en el supuesto de que no se operara una clara delimitación y coordinación de las tareas y funciones entre los diversos niveles territoriales, que evitara solapamientos, duplicidades y eventuales conflictos competenciales, así como inviable en caso de no concretarse cómo se financiarían cada una de las entidades territoriales.

Las decisiones se adoptan y las políticas se diseñan desde el centro, lo que resulta escasamente eficiente. Además en aras de la protección del modelo unitario y del concepto de

En la base encontramos los municipios, los distritos y los territorios indígenas; en el escalón inmediatamente superior, las provincias; por encima de éstas, los departamentos; más arriba, las regiones y, por último, la Nación. soberanía, la Nación ha interferido en el ámbito competencial de las entidades territoriales, lo que ha generado una confusión en materia de competencias que se traduce en una duplicación de funciones (Restrepo, 2006).

En este marco según Jacint Jordana la situación de la descentralización en Colombia nos muestra la existencia de un pluralismo institucional muy intenso, aunque con un fuerte predominio de vetos o imposiciones para articular las relaciones entre niveles, mientras que el monopolio institucional distribuido es casi inexistente. Así, es frecuente, especialmente en las áreas sociales, que participen los tres niveles de gobierno en forma interrelacionada en una misma política, existiendo diversas fórmulas de relaciones intergubernamentales que actúan simultáneamente (fiscales, evaluación, normativas, etc.). Por lo anterior es muy importante resaltar que en el proceso de descentralización que empezó Colombia, es necesario generar un fortalecimiento de instancias de coordinación para la formulación e implementación de políticas públicas que ayuden a legitimar el proceso descentralizador y con ello se fortalezca la Gobernanza, mediante la participación activa y efectivas de otros actores en la toma de decisiones. Con ello se garantizará que se tomen soluciones efectivas a las problemáticas de las diferentes comunidades que convergen en el país; pero es imprescindible precisar que es necesario la regulación normativa de las instancias intergubernamentales y por otro lado la configuración de cuadros con conocimiento en el campo de la gestión pública que coadyuguen al fortalecimiento institucional y con ello al desarrollo democrático y participativo del país. 


\section{Conclusiones}

1. La forma concreta que adopte la descentralización en cada país, está influida por sus contextos sociopolíticos, y económicos que a corto plazo delimitan el papel específico de los mecanismos y reglas institucionales. De ahí que cada proceso de descentralización es esencialmente único y específico de cada país.

2. Las relaciones intergubernamentales 0 instancias de coordinación son esenciales para legitimar el proceso de descentralización y el fortalecimiento de la Gobernanza en un país.

3. En Colombia es importante desarrollar un estricto marco jurídico, financiero y administrativo para facilitar el desarrollo de los mismos, esto ha hecho que no haya competencias delimitadas y con ello que haya superposición de tareas, conflictos y baja eficacia, eficiencia y legitimidad en el accionar público llevando a una baja Gobernanza y gobernabilidad institucional.

4. El fortalecimiento o profundización del proceso de descentralización en Colombia debe ser coherente a las realidades y circunstancias del país, con ritmos y esquemas diferentes en la asignación de competencias a las entidades territoriales, evaluando impactos, y corrigiendo las desviaciones que se pueden generar en el proceso y coherente entre los objetivos propuestos e instrumentos utilizados.

\section{Referencias}

Agranoff Robert (1995). Marcos para el análisis comparado de las relaciones intergubernamentales. Madrid: Papeles de trabajo Gobierno y Administración pública, Instituto Universitario Ortega y Gasset.

Agranoff, Robert (1997). Las relaciones y la Gestión Intergubernamental. en Bañón R. Y Carrillo, E. La nueva Administración Pública. Madrid: Alianza .

Aja, E. (1999). El Estado Autonómico. Federalismo y hechos diferenciales, Madrid: Alianza.

Anderson William. (1960). Interguvermental relations in review. Minneapolis: University of Minnesota Press.

Arana Muñoz J. (2007). Gobierno y pacto local. La incidencia de la carta europea de la autonomía local en la reforma del régimen local español.

Boisier S. (1990). La descentralización: un tema difuso y confuso. Serie Ensayos, Documento 90/05. Santiago de Chile: Ilpes.

Castro Jaime, (2003). La cuestión territorial, 2ª ed., Bogotá: Editorial Oveja Negra.

Camou, A (2001). Estudio preliminar, en Camou A. Los desafíos de la gobernabilidad, México: Flacso, Plaza y Valdés.

Cohen, J Y Peterson. Stephen B. (1997). Administrative Decentralization: a New Framework for Improved Governance, Accountability, and Performance, Development Discussion Paper 582, Harvard Institute for International Development.

Constitución Política De Colombia.

Corte Constitucional, Sentencia C-346 de 1997.

Cruz. C. N. (2001). Gobernabilidad y «governance» democráticas: El confuso y no siempre evidente vínculo conceptual e institucional. Revista electrónica Dhial, Instituto Internacional de Gobernabilidad www.iigov.org/ dhial/?p=23 05. 
Girón Resgueira E. (2007). La forma de Estado en la Constitución Política de 1991 En García Ruíz J. ¿hacia donde se debe orientar la organización del territorio en Colombia? Bogotá: Universidad libre de Colombia.

Illán Sailer J. (2006). Los Procesos de Descentralización y los retos para la ayuda internacional Fiiap. Madrid: Fundación internacional para Iberoamérica de Administración y Políticas Públicas ed. Cyan.

Jordana J. (2001). Documentos de trabajo proyecto conjunto Indes-Union Europea: Relaciones intergubernamentales y descentralización en América Latina: una perspectiva institucional. Departamento de integración y programas regionales. Instituto Interamericano para el Desarrollo Social. Banco Interamericano De Desarrollo. Serie de Documentos de Trabajo I-22 UE

Legales P. (1998). Régulation, gouvernance et territoire, en Commaille, J. y Jobert, B. (dirs.): La régulation politique à paraître, Paris: Presses de Sciences Po.

Mayntz R. (2000). Nuevos desafíos de la teoría de Governance, Revista instituciones y desarrollo Vol, 7.

Olías De Lima B, Del Campo E. (2008). Buen gobierno, rendimiento institucional y participación en las democracias contemporáneas. Revista Sistema N. 203-204. Madrid

Olías De Lima, Blanca. (2001). La evolución de la gestión pública: La nueva Gestión Pública. En La nueva Gestión pública coord., Blanca Olías de Lima. México: Ed. Prentice Hall.

Planeación Nacional (2008). Descentralización y Gobierno Intermedios locales, tres experiencias internacionales Bogotá.
Prats J. O. (2002) y Cruz (2001). El concepto y el análisis de la gobernabilidad. Revista electrónica Instituciones y Desarrollo, № 14-15. Instituto Internacional de Gobernabilidad. 2003. http://www.iigov.org/revista/?p=14 08.

Ramio, Charles (2001) Los Problemas de la Implantación de la Nueva Gestión Pública en las Administraciones Públicas Latinas: Modelo de Estado y Cultura institucional. Revista del CLAD: Reforma y Democracia № 21, Caracas, Venezuela.

Rhodes Aw. Y Wright V. (1997) Introduction, tensions in the territorial politics of Western Europe Londres. en Agranoff, R. Las relaciones y la Gestión Intergubernamental En Bañón R. Carrillo E. La nueva Administración Pública.Madrid: .Alianza.

Restrepo Botero D. (2007). Paradoja de la descentralización ed. Red de Iniciativas para la Gobernabilidad, la Democracia y el Desarrollo Territorial Bogotá: RINDE .

Rose R, (1995), Understanding Big Government. En Agranoff, R Marcos para el análisis comparado de las relaciones intergubernamentales. papeles de trabajo Gobierno y Administración pública, Instituto Universitario Ortega y Gasset, Madrid.

Ruano De La Fuente J. M, (2002). La Gobernanza como forma de acción pública y como concepto analítico. VII Congreso Internacional del CLAD sobre la Reforma del Estado y de la Administración Pública, Lisboa, Portugal.

Wright D. (1997). Para entender las Relaciones Intergubernamentales. Colegio Nacional de Ciencias Políticas y Administración Pública. México: Fondo de Cultura Económica de México. 